\title{
A NOTE ON THE JOINT OPERATOR NORM OF HERMITIAN OPERATORS ON BANACH SPACES
}

\author{
by MUNEO CHŌ† and TADASI HURUYA
}

(Received 20 February, 1991)

Let $X$ be a complex Banach space and $H$ be a hermitian operator on $X$. Then in [7] Sinclair proved that $r(H)=\|H\|$, where $r(H)$ and $\|H\|$ are the spectral radius and the operator norm of $H$, respectively.

For a commuting $n$-tuple $\mathbf{T}=\left(T_{1}, \ldots, T_{n}\right)$ of operators on $X$, we denote the (Taylor) joint spectrum of $\mathbf{T}$ by $\sigma(\mathbf{T})$ (see [9]) and define the joint operator norm $\|\mathbf{T}\|$ and the joint spectral radius $r(\mathbf{T})$ by

$$
\|\mathbf{T}\|=\sup _{\|x\|=1}\left(\sum_{i=1}^{n}\left\|T_{i} x\right\|^{2}\right)^{1 / 2}
$$

and

$$
r(\mathbf{T})=\sup \{|z|: z \in \sigma(\mathbf{T})\}
$$

respectively.

When $X$ is a Hilbert space, it holds that $r(\mathbf{T})=\|\mathbf{T}\|$ for a doubly commuting $n$-tuple $\mathbf{T}=\left(T_{1}, \ldots, T_{n}\right)$ of hyponormal operators (see [3]). We asked in [2] whether the equality $r(\mathbf{H})=\|\mathbf{H}\|$ holds for a commuting $n$-tuple $\mathbf{H}$ of hermitian operators on any Banach space. In this paper we will give an answer to this problem.

EXAmple. Let $X=B\left(\mathbb{C}^{3}\right)$ (the set of all 3 by 3 matrices with the operator norm). Let

$$
A=\left[\begin{array}{rrr}
1 & 0 & 0 \\
0 & -\frac{1}{2} & 0 \\
0 & 0 & -\frac{1}{2}
\end{array}\right] \text { and } B=\left[\begin{array}{rrr}
0 & 0 & 0 \\
0 & \frac{\sqrt{3}}{2} & 0 \\
0 & 0 & -\frac{\sqrt{3}}{2}
\end{array}\right] \text {. }
$$

For $S \in X$ let $D_{S}$ denote the derivation on $X$ defined by $D_{S}(T)=S T-T S$. Then $\mathbf{H}=\left(D_{A}, D_{B}\right)$ is a commuting pair of hermitian operators satisfying $r(\mathbf{H})<\|\mathbf{H}\|$.

Proof. It is easy to see that $\mathbf{H}$ is a commuting pair of hermitian operators (see [6]). Also we have

$$
\sigma(\mathbf{H})=\left\{(0,0),\left(\frac{3}{2}, \pm \frac{\sqrt{3}}{2}\right),\left(-\frac{3}{2}, \pm \frac{\sqrt{3}}{2}\right),(0, \pm \sqrt{3})\right\} .
$$

Hence we have $r(\mathbf{H})=\sqrt{3}$.

Next let

$$
T=\left[\begin{array}{lll}
0 & 0 & 1 \\
1 & 0 & 0 \\
0 & 1 & 0
\end{array}\right]
$$

† This research was partially supported by Grant-in-Aid Scientific Research.

Glasgow Math. J. 34 (1992) 219-220. 
Since then $\|T\|=1$,

$$
D_{A}(T)=\left[\begin{array}{rrr}
0 & 0 & \frac{3}{2} \\
-\frac{3}{2} & 0 & 0 \\
0 & 0 & 0
\end{array}\right] \text { and } D_{B}(T)=\left[\begin{array}{ccc}
0 & 0 & \frac{\sqrt{3}}{2} \\
\frac{\sqrt{3}}{2} & 0 & 0 \\
0 & -\sqrt{3} & 0
\end{array}\right] \text {, }
$$

we have $\left\|D_{A}(T)\right\|^{2}+\left\|D_{B}(T)\right\|^{2}=\frac{9}{4}+3=\frac{21}{4}>4$. Hence it holds that

$$
r(\mathbf{H})=\sqrt{3}<2<\|\mathbf{H}\| \text {. }
$$

\section{REFERENCES}

1. F. F. Bonsall and J. Duncan, Numerical ranges II (Cambridge Univ. Press, 1973).

2. M. Chō, Joint spectra of commuting normal operators on Banach spaces, Glasgow Math. J. 30 (1988), 339-345.

3. M. Chō and M. Takaguchi, Some classes of commuting $n$-tuple of operators, Studia Math. 80 (1984), 245-259.

4. $M$. Chō and $H$. Yamaguchi, A simple example of a normal operator $T$ such that $r(T)<\|T\|$, Proc. Amer. Math. Soc. 108 (1990), 143.

5. M. Rosenblum, On the operator equation $B X-X B=Q$, Duke Math. J. 23 (1956), $263-269$.

6. S.-Y. Shaw, On numerical ranges of generalized derivations and related properties, $J$. Australian Math. Soc. (A) 36 (1984), 134-142.

7. A. M. Sinclair, The norm of a hermitian element in a Banach algebra, Proc. Amer. Math. Soc. 28 (1971), 446-450.

8. J. G. Stampfli, The norm of a derivation, Pacific J. Math. 33 (1970), 737-747.

9. J. L. Taylor, A joint spectrum for several commuting operators, J. Functional Anal. 6 (1970), 172-191.

Department of Mathematics

JoETSU UNIVERSITY OF EDUCATION JOETSU 943, JAPAN
FACUlTy OF Education

NigGata UNIVERSITY

NiIgata 950-21, JAPAN 